\title{
THE ISOPERIMETRIC INEQUALITY FOR NONSIMPLE CLOSED CURVES
}

\author{
LILIANA M. GYSIN
}

(Communicated by Palle E. T. Jorgensen)

\begin{abstract}
The main purpose of this paper is the generalization to the hyperbolic and elliptic spaces of the isoperimetric inequalilty of Banchoff and Pohl (J. Differential Geom. 6 (1971), 175-192).
\end{abstract}

1

Banchoff and Pohl [1] showed that the classical isoperimetric inequality still holds for nonsimple closed plane curves of length $L$, if the area is replaced by the sum of the areas into which the curve divides the plane, each weighted with the square of the winding number, i.e.,

$$
L^{2}-4 \Pi \int_{\mathbb{R}^{2}} w^{2} d P \geq 0 .
$$

Equality holds only for one or several coincident circles, each traversed in the same direction a number of times. The authors also generalized the result to arbitrary dimension and codimension.

In this paper we show that a similar result is valid for compact oriented manifolds immersed in either the hyperbolic or the elliptic space. In $\S 2$ we obtain a generalization of formulae (4.7) and (4.8) of [1] in any $n$-dimensional Riemann-space of constant curvature by using some known formulae of integral geometry [3]. In $\S 3$ we show that the results of Banchoff and Pohl [1, $\S 3]$ are valid without change in the hyperbolic and elliptic $n$-dimensional spaces. In $\S 4$ we obtain a general expression for the main result of Banchoff and Pohl [1], which can be stated as:

Let $\mathbb{W}^{n}$ denote the hyperbolic, elliptic, or euclidean $n$-dimensional space with $H(r)$ being $\operatorname{sh} r$, sen $r$, or $r$, respectively. Let $M$ be a compact oriented manifold of dimension $m$ and $f: M \rightarrow \mathbb{W}^{n}$ be an immersion of class $C^{2}$. For $(x, y) \in M \times M$ let $r(x, y)$ denote the chord length from $f(x)$ to $f(y)$ and let $d V_{1}, d V_{2}$ denote the volume elements on $M$ at $x$ and $y$, respectively. Let $H_{n-m-1, n}$ denote the Grassmann manifold of all (unoriented) $(n-m-1)$ planes in $\mathbb{W}^{n}$ (parallel planes are not identified), and let $\left|d H_{n-m-1, n}\right|$ denote its invariant measure. For $h \in H_{n-m-1, n}$ let $\pm \lambda$ denote the linking number of

Received by the editors November 12, 1990.

1980 Mathematics Subject Classification (1985 Revision). Primary 53A35. 
$h$ with $f$. Then:

$$
\begin{gathered}
\int_{M \times M} H(r)^{-m+1} d V_{1} d V_{2}-(1+m) \Sigma_{m} K_{m, n} \int_{H_{n-m-1, n}} \lambda^{2}\left|d H_{n-m-1, n}\right| \\
+\frac{(-1)^{m(m+1) / 2}}{m !} \int_{G \cap M \times M \neq \varnothing}[H(r)-r] d G^{m} \geq 0,
\end{gathered}
$$

where $\Sigma_{m}$ denotes the surface volume of the unit $m$-sphere and $K_{m, n}$ is a constant, which depends only on $m$ and $n$. Equality holds only for one or several coincident spheres with coincident orientations or $(m=1)$ one or several circles each traversed in the same direction a number of times.

For closed nonsimple plane curves we obtain

$$
L^{2}-4 \Pi \int_{\mathbf{W}^{2}} w^{2} d P-\int_{G \cap(M \times M)}[H(r)-r] d \vec{G} \geq 0,
$$

where $d \vec{G}$ denotes the density for oriented geodesics.

This formula, when applied to simple closed curves, taking into account (18.10) and (18.11) of [3], gives the classical isoperimetric inequality:

$$
L^{2}-4 \Pi F+\varepsilon F^{2} \geq 0,
$$

where $\varepsilon$ equals $-1,1$, or 0 for the hyperbolic, elliptic, or euclidean plane, respectively.

Let $\mathbb{W}^{n}$ be a Riemann manifold of dimension $n$ endowed with the fundamental quadratic form (for a local coordinate system) (see [3, p. 331])

$$
d s^{2}=\sum_{i, j=1}^{n} g_{i j} d x_{i} d x_{j}
$$

Since the 2-form

$$
d G=\sum_{i=1}^{n} d p_{i} \wedge d x_{i}
$$

is an invariant form (any geodesic is defined by a point $\left(x_{1}, \ldots, x_{n}\right)$ and a direction $\left.\left(p_{1}, \ldots, p_{n}\right)\right)$, the exterior powers

$$
d G^{m}=(d G)^{m}=m ! \sum_{i_{1}<\cdots<i_{m}} d p_{i_{1}} \wedge d x_{i_{1}} \wedge \cdots \wedge d p_{i_{m}} \wedge d x_{i_{m}}
$$

are also invariant forms $(m=1, \ldots, n-1)$ and they define a measure for $2 m$-dimensional sets of geodesics. Consider the expression:

$$
d G^{m}=m !(-1)^{m(m-1) / 2} \sum_{i_{1}<\cdots<i_{m}} d p_{i_{1}} \wedge \cdots \wedge d p_{i_{m}} \wedge d x_{i} \wedge \cdots \wedge d x_{i_{m}}
$$

for a family of geodesics parametrized in a special way; in particular, for geodesics that join the points of one $m$-dimensional submanifold of $\mathbb{W}^{n}$ to another. We restrict ourselves to $\mathbb{W}^{n}$ the $n$-dimensional hyperbolic, elliptic, or euclidean space, noting that they include any complete, simply-connected Riemann-space with constant curvature. 
Let us consider as Pohl [2, p.1325] the following slightly more general situation:

Let $M_{1}, M_{2}$ be oriented $m$-dimensional differentiable manifolds of class $C^{1}$. Let $f: M_{1} \rightarrow \mathbb{W}^{n}$ be an immersion, and let $F: M_{1} \times M_{2} \rightarrow \mathbb{W}^{n}$ be a continuously differentiable map such that for each fixed $x \in M_{1}$ the map $F_{x}(y)=F(x, y)$ describes an immersion of $M_{2}$. We assume as Pohl that $F(x, y) \neq f(x) \forall x, y$. Let $G$ denote the geodesic that joins $f(x)$ to $F(x, y)$ oriented from $f(x)$ to $F(x, y)$. We are now going to prove equation (2.5) of [2] under these premises. For this purpose, let us define orthonormal frames $X e_{1}, \ldots, e_{n}$ and $X a_{1}, \ldots, a_{n}$ following Pohl [2, p.1325] with only the following modifications:

$e_{1}$ is the tangent unit vector to $G$ in $f(x)$;

$S_{1}$ contains $T_{1}$ and $e_{1}$ (but not $G$ ); and

$U_{1}$ is the $(m+1)$-dimensional manifold containing $M_{1}$ and $G$, so that

$S_{1}$ is the tangent space to $U_{1}$ in $f(x)$.

Since the orientations $e_{1} e_{2}$ and $a_{1} a_{2}$ agree on $Q_{1}$, we may write

$$
d e_{2}=\operatorname{sen} \sigma_{1} d a_{2} \text {. }
$$

We now determine $G$ in $U_{1}$ by its intersection with $S^{\prime}\left(e_{1}=0\right) \subset U_{1}$, i.e., $X$. Following Santaló [3, p. 334] we have:

$$
d s^{2}=\sum_{i=1}^{n} g_{i i} d x_{i}^{2}, \quad p_{i}=\left(g_{i i}\right)^{1 / 2} \cos \alpha_{i}, \quad \alpha_{i}=\angle\left(G, e_{i}\right) .
$$

Using expression (2.1)

$$
d G^{m}=m !(-1)^{(m-1) m / 2} d p_{2} \wedge \cdots \wedge d p_{m+1} \wedge d e_{2} \wedge \cdots \wedge d e_{m+1} .
$$

Also

$$
d u_{m}=\operatorname{sen} \alpha_{2} \cdots \operatorname{sen} \alpha_{m+1} d \alpha_{2} \wedge \cdots \wedge d \alpha_{m+1},
$$

where $d u_{m}$ denotes the area element on the unit $m$-dimensional sphere of center $X$ corresponding to the direction of $e_{1}$ [3, p. 337] (notice that $\alpha=$ $\left.\angle\left(e_{1}, e_{1}\right)\right)$. Since the volume element $d V_{1}$ on $M$ at $X$ has the value:

$$
d V_{1}=\left(g_{22} \cdots g_{m+1 m+1}\right)^{1 / 2} d a_{2} \wedge \cdots \wedge d a_{m+1},
$$

replacing (2.2), (2.3), (2.5), and (2.6) in (2.4) we obtain

$$
d G^{m}=m !(-1)^{m(m-1) / 2} \operatorname{sen} \sigma_{1} d u_{m} \wedge d V_{1} .
$$

Next we compute $d u_{m}$ with fixed $x$. To do this we translate the frame $X e_{1}$, $\ldots, e_{n}$ parallel along $G$ to $F_{x}(y)$ obtaining $X^{\prime} e_{1}^{\prime}, \ldots, e_{n}^{\prime}$. Let $S_{2}$ be the $(m+1)$-dimensional linear space through $X^{\prime}$ containing $e_{1}^{\prime}$ and $T_{2}$, the tangent space to $F_{x}$ at $y$. We orient $S_{2}$ by $e_{1}^{\prime} T_{2}$ unless $e_{1}^{\prime}$ lies in $T_{2}$, in which case we take either orientation on $S_{2}$. Let $\sigma_{2}=\angle\left(T_{2}, e_{1}^{\prime}\right), X^{\prime} b_{1}, \ldots, b_{n}$ be an orthonormal frame such that $b_{1}=e_{1}^{\prime} ; b_{2}, \ldots, b_{m+1}$ are in $S_{2}$ and so chosen that $b_{1} \cdots b_{m+1}$ agrees with the given orientation of $S_{2}$.

We use polar coordinates in $S_{2}$, with respect to the frame $X b_{1}^{\prime}, \ldots, b_{n}^{\prime}$ (where $b_{i}^{\prime}$ is the parallel translated vector of $b_{i}$ from $X^{\prime}$ to $X$ along $G$ ). Therefore, we have

$$
d s^{2}=d r^{2}+H(r) d \lambda_{i}^{2}
$$


and for fixed $r$,

$$
d b_{i}=H(r) d \lambda_{i}, \quad d \sigma_{p}=H(r)^{m} d u_{m}^{\prime},
$$

where $d \sigma_{p}$ denotes the $m$-dimensional volume element for fixed $r$, and $d u_{m}^{\prime}$ is the area element on the unit $m$-dimensional euclidean sphere of center $X$ corresponding to the direction of $b_{1}^{\prime}=e_{1}$ (defined for the frame $b_{1}^{\prime}, \ldots, b_{n}^{\prime}$ ).

On the other hand if $d V_{2}$ denotes the volume element on $M_{2}$ at $X^{\prime}$,

$$
d \sigma_{p}=d V_{2} \operatorname{sen} \sigma_{2}
$$

and if we define $\cos \tau$ as Pohl [2, p. 1326] so that $\tau$ is interpreted as the angle between the oriented $(m+1)$-planes $S_{1}$ and $S_{2}$ (or its parallel translation along $G)$ we have

$$
\cos \tau=d u_{m} / d u_{m}^{\prime}
$$

so that

$$
d u_{m}=H(r)^{-m} \cos \tau \operatorname{sen} \sigma_{2} d V_{2} .
$$

Replacing in (2.7) we obtain

$$
d G^{m}=m !(-1)^{m(m-1) / 2} \operatorname{sen} \sigma_{1} \operatorname{sen} \sigma_{2} \cos \tau H(r)^{-m} d V_{2} \wedge d V_{1},
$$

which is one of the results of Pohl [2, p. 1326].

In order to obtain the general result we have to compute

$$
\int_{M \times M} r d G^{m}=\int_{M \times M}\left(r d p_{2}^{\prime}\right) \wedge d a_{2} \wedge d G^{m-1},
$$

where $X a_{1}, \ldots, a_{n}$ is the frame defined above so that $p_{2}^{\prime}=\left(g_{22}\right)^{1 / 2} \cos \sigma_{1}$. To do this we define as in [1] a certain 'secant space' and apply Stoke's theorem:

$$
\int_{M \times M} r d p_{2}^{\prime}=\left.r \cdot p_{2}^{\prime}\right|_{r=0}-\int_{M \times M} p_{2}^{\prime} d r=-\int\left(g_{22}\right)^{1 / 2} \cos \sigma_{1} d r
$$

Since $\cos \sigma_{2}=d r / d b_{2}$ (for $X b_{1}, \ldots, b_{n}$ the frame defined above) we get:

$$
\int_{M \times M} r d G^{m}=-\int_{M \times M}\left(g_{22}\right)^{1 / 2} \cos \sigma_{1} \cos \sigma_{2} d b_{2} \wedge d a_{2} \wedge d G^{m-1} .
$$

Defining frames as above and interpreting the angle $\nu$ as in [1] we have

$$
d u_{m-1}=\cos \nu d u_{m-1}^{\prime}
$$

and therefore,

$$
\begin{aligned}
d G^{m-1}= & (m-1) !(-1)^{m(m-1) / 2} \cos \nu\left(g_{33} \cdots g_{m+1 m+1}\right)^{1 / 2} d u_{m-1}^{\prime} \\
& \wedge d a_{3} \wedge \cdots \wedge d a_{m+1} .
\end{aligned}
$$

Since

and

$$
d V_{1}=\left(g_{22} \cdots g_{m+1 m+1}\right)^{1 / 2} d a_{2} \wedge \cdots \wedge d a_{m+1}
$$

replacing in $(2.10)$

$$
d V_{2}=H(r)^{-m+1} d b_{2} \wedge d u_{m-1}^{\prime},
$$

$$
\begin{aligned}
\int_{M \times M} r d G^{m}= & -(m-1) !(-1)^{m(m-1) / 2} \\
& \times \int_{M \times M} H(r)^{-m+1} \cos \sigma_{1} \cos \sigma_{2} \cos \nu d V_{2} \wedge d V_{1},
\end{aligned}
$$

which is the desired expression. 
We now prove that the formulae of Banchoff and Pohl $[1, \S 3]$ are valid without change in the elliptic and hyperbolic $n$-dimensional spaces. For this purpose we work in an analogous way to Banchoff and Pohl [1] but we make use of some known results of integral geometry [3].

We first consider the second integral in our result (see (1.1)) and write

$$
\mathscr{A}(M)=K_{m, n} \int_{H_{n-m-1, n}} \lambda^{2}\left|d H_{n-m-1, n}\right|
$$

where

$$
K_{m, n}=\frac{\Sigma_{n-m-1} \cdots \Sigma_{1}}{\Sigma_{n} \cdots \Sigma_{m+2}} \quad(n \geq m+2), K_{m, m+1}=1,
$$

is defined in Banchoff and Pohl [1, p. 180]. We now prove that $\mathscr{A}(M)$ is stable under rising of the codimension, i.e., if $f(M) \subseteq \mathbb{W}^{n} \subseteq \mathbb{W}^{N}$ then

$$
K_{m, N} \int_{H_{N-m-1, N}} \lambda^{2}\left|d H_{N-m-1, N}\right|=K_{m, n} \int_{H_{n-m-1, n}} \lambda^{2}\left|d H_{n-m-1, n}\right| .
$$

Comparing [3, (14.69)] firstly for $q \rightarrow n, r \rightarrow n-m, n \rightarrow n+1, M_{q} \rightarrow U_{n}$, and secondly for $q \rightarrow n, r \rightarrow n-m-1, n \rightarrow n, M_{q} \rightarrow U_{n}$, we obtain

$$
\int_{H_{n-m, m+1} \cap U_{n} \neq \varnothing} d H_{n-m, m+1}=\frac{\Sigma_{n+1}}{\Sigma_{n-m}} \int_{H_{n-m-1, n} \cap U_{n} \neq \varnothing} d H_{n-m-1, n} .
$$

On the other hand (see [2] or [3]),

$$
\begin{aligned}
d H_{n-m, n+1}=\bigwedge_{j, \beta} \omega_{j \beta} \wedge \omega_{\beta} & \\
(j=1, \ldots, n-m ; \beta=n-m+1, \ldots, n+1), & \\
d H_{n-m-1, n}=\bigwedge_{i, \alpha} \pi_{i \alpha} \wedge \pi_{\alpha} & \quad(i=1, \ldots, n-m-1 ; \alpha=n-m, \ldots, n)
\end{aligned}
$$

with $\omega_{j \beta}=d e_{\beta} \cdot e_{j}, \omega_{\beta}=d p \cdot e_{\beta}, \omega_{j \beta}+\omega_{\beta j}=0$, for an orthonormal frame $p e_{1}, \ldots, e_{n+1}$ in $p$, and $\pi_{i \alpha}=d a_{\alpha} \cdot a_{i}, \pi_{\alpha}=d p \cdot a_{\alpha}, \pi_{i \alpha}+\pi_{\alpha i}=0$ for $p a_{1}, \ldots, a_{n+1}$ the frame defined as follows:

$$
\begin{array}{cc}
a_{i}=e_{i}, \quad i=1, \ldots, n-m-1 ; & a_{n-m}=e_{n+1} ; \\
a_{n-m+1}=e_{n-m+1}, \quad i=1, \ldots, m ; & a_{n+1}=e_{n-m} ;
\end{array}
$$

therefore,

$$
\left|d H_{n-m, n+1}\right|=\left|d H_{n-m-1, n}\right| \wedge \bigwedge_{\alpha} \pi_{n+1, \alpha}, \quad \alpha=n-m, \ldots, n .
$$

Since the linking number of an $(n-m)$-plane $h \subseteq \mathbb{W}^{n+1}$ with $M$ does not change regarding $h$ as lying in $\mathbb{W}^{n}$, using Fubini's theorem and integrating $\lambda^{2}$ we get

$$
\int_{H_{n-m, n+1}} \lambda^{2}\left|d H_{n-m, n+1}\right|=c_{n, m} \int_{H_{n-m-1, n}} \lambda^{2}\left|d H_{n-m-1, n}\right| .
$$

Applying (3.3) we obtain $c_{n, m}=\Sigma_{n+1} / \Sigma_{n-m}$. This proves the assertion for $N=n+1$, and so (3.2) is valid by induction. 
Also since both the Cauchy formula and the remaining arguments used by Banchoff and Pohl [1] to demonstrate:

$$
\mathscr{A}\left(M^{0}\right)=K_{0, n} \int_{H_{n-1, n}} \lambda^{2}\left|d H_{n-1, n}\right|=-1 / 2 \Sigma_{i, j} r\left(x_{i}, x_{j}\right) i_{i} i_{j}
$$

are also valid in the hyperbolic and elliptic spaces, equation (3.4) stays valid without change.

In order to prove that for $q>n-m-1$

$$
\mathscr{A}\left(M^{m}\right)=1_{m, n, q} \int_{H_{q, n}} \mathscr{A}\left(M^{m} \cap H_{q}\right)\left|d H_{q, n}\right|
$$

with

$$
1_{m, n, q}=\frac{K_{m, n} \Sigma_{q-n+m} \cdots \Sigma_{0} \Sigma_{n-q-1} \cdots \Sigma_{0}}{K_{q-n+m, q} \Sigma_{m} \cdots \Sigma_{0}},
$$

we use the fact that equations (12.36) and (12.52) of [3] are also valid in hyperbolic and elliptic spaces, so that

$$
\begin{gathered}
\int_{\text {total }} d H_{r, n}[q] \\
d H_{i+1, r} \wedge d H_{r, n}^{*}=d H_{r, n^{[i+1]}} \wedge d H_{i+1, n},
\end{gathered}
$$

where $d H_{r, n^{[i+1]}}$ is the density of $H_{r, n}$ about $H_{i+1, r}$ and $H_{r, n}^{*}$ denote the oriented Grassmannians. Using that, for

$$
H_{n-m-1, q} \subseteq H_{q, n},
$$

it is

$$
\lambda\left(M^{m}, h_{n-m-1}\right)=\lambda\left(M^{m} \cap h_{q}, h_{n-m-1}\right),
$$

we now integrate $\lambda^{2}$ over the set of pairs of linear spaces $\left(H_{q, n} ; H_{n-m-1, q}\right)$ so that (3.8) is valid, and apply (3.7) to obtain

$$
\int \lambda^{2} d H_{n-m-1, q} \wedge d H_{q, n}^{*}=\int \lambda^{2} d H_{q, n^{[n-m-1]}} \wedge d H_{n-m-1, n} .
$$

Evaluating the right-hand side using equation (3.6) for $r \rightarrow q, q \rightarrow n-m-1$; and taking into account the definition (3.1) in both sides, we compare and get equation (3.5) after few calculations.

Comparing as above, the densities $d H_{n-m, n}$ and $d H_{n-m-1, n-1}$, using orthonormal frames $p e_{1}, \ldots, e_{n}$ and $p a_{1}, \ldots, a_{n}$ with $a_{1}=e_{1} ; a_{i}=e_{n-m-1+i}$ $i=2, \ldots, m+1 ; a_{m+j}=e_{j} j=2, \ldots, n-m$; and using:

$$
\left|d G^{m}\right| / m !=\left|\pi_{12} \wedge \cdots \wedge \pi_{1 m+1} \wedge \pi_{2} \wedge \cdots \wedge \pi_{m+1}\right|
$$

(which is easy to prove from expression (2.1)), we obtain

$$
\left|d H_{n-m, n}\right|=\left|d H_{n-m-1, n-1[0]}\right| \wedge(1 / m !)\left|d G^{m}\right| .
$$

We now integrate the function $f(r)=r$ using Fubini's theorem to get

$$
\int r\left|d H_{n-m, n}\right|=c_{m, n} / m ! \int r\left|d G^{m}\right| \text {. }
$$


Using a general result of differential geometry (see [2, p. 1328]) and a particular case of (3.5) combined with (3.4), and evaluating the constant $c_{m, n}$ as Banchoff and Pohl [1, p. 186], equation (3.9) yields

$$
\mathscr{A}\left(M^{m}\right)=\frac{(-1)^{1+m(m+1) / 2}}{m ! \Sigma_{m}} \int_{M \times M} r\left(d G^{m}\right),
$$

which is Theorem 4 of [1].

In order to obtain the general result we are now able to use the same arguments as Banchoff and Pohl [1] with $H(r)$ instead of $r$. Using the results of $\S \S 2$ and 3 , we obtain

$$
\int H(r)^{-m+1} d V_{1} d V_{2}-(1+m) \Sigma_{m} \mathscr{A}(M)+\frac{(-1)^{m(m+1) / 2}}{m !} \int[H(r)-r] d G^{m} \geq 0
$$

with equality holding for one or several coincident spheres with coincident orientations or for $(m=1)$ one or several coincident circles all transversed in the same direction each a number of times. Applied to the euclidean space $(H(r)=r)$, it yields the main theorem of Banchoff and Pohl [1].

\section{REFERENCES}

1. T. F. Banchoff and W. F. Pohl, A generalization of the isoperimetric inequality, J. Differential Geom. 6 (1971), 175-192.

2. W. F. Pohl, Some integral formulas for space curves and their generalization, Amer. J. Math. 90 (1968), 1321-1345.

3. L. A. Santaló, Integral geometry and geometric probability, Encyclopedia Math. Appl., vol. 1, Addison-Wesley, Reading, MA, 1976.

Departamento de Matemática, Facultad de Ciencias Exactas y Naturales, Universidad de Buenos Aires, Ciudad Universitaria (1428) Capital Federal, Argentina

E-mail address: lgysin@mate.dcfcen.edu.ar 